\title{
Chapter XII
}

\section{Usable and Interoperable \\ E-Learning Resources Repositories}

\author{
S. Retalis \\ University of Piraeus, Greece
}

\section{Abstract}

The Web puts a huge number of learning resources within reach of anyone with Internet access. In many cases, these valuable resources are difficult for most users to find in an efficient and effective manner. What makes an e-learning resources repository much more than a portal is the ability to discover a learning object and put it to a new use. The purpose of an elearning resources repository is not simply safe storage and delivery but the ability of their administration, in terms of updating, identifying, utilizing, sharing and re-using them, which remains a great challenge. Moreover, the various repositories are either closed systems or systems that allow user access only through proprietary interfaces and data formats. In brief, there 
is lack of interoperability. The aim of this chapter is to present the requirements of an ideal e-learning resources repository that will provide services for covering the aforementioned critical issues. We will also describe such an ideal system could be non-centralized, which is the main difference from all the system that exists today in the WWW. Peer to Peer $(P 2 P)$ based approaches are more flexible than centralized approaches with several advantages.

\section{Introduction}

The Web puts a huge number of learning resources within reach of anyone with Internet access. One can mention a lot of Web sites that hold learning resources, such as Canada's SchoolNet (http://www.schoolnet.ca/), MathGoodies (http:// www.mathgoodies.com), or the U.S.-based site maintained by the Educational Object Economy Foundation (http://www.eoe.org/), and many more. The National Governors Association in the United States published a report in 2001 mentioning that " $58 \%$ of all two- and four-year colleges offered distance learning courses in 1998, while $84 \%$ of all colleges expected to do so by 2002" (NGA, 2002). As the number of Web sites continues to grow, search engine retrieval effectiveness is likely to decline, and there is a need to consider alternative resource discovery mechanisms (Milstead \& Feldman, 1999).

Apart from the "discovery" problem, the learning resource sharing appears as a major challenge and necessity, because development costs are becoming significant (Zlomislic \& Bates, 2002). Since the old days, educators have been reusing learning resources. Textbooks, wall maps in geography classes, periodic tables of the elements in science classes, filmstrips and videos, etc., are resources that appear in many $\mathrm{K}-12$ classrooms worldwide (Downes, 2001). Nowadays, coming into the e-learning era, educators and learners need to have access to as well as to reuse e-learning resources of their interests, needs, and preferences.

This is why e-learning resources repositories or e-Learning Resources Brokerage Systems (LRBS) have emerged. In very generic terms, an online "brokerage system" is an online entity that acts as a one-stop electronic marketplace. A brokerage system has two types of users: those who offer their products for sale (providers) and those who buy the products offered (consumers). An e-learning objects brokerage system facilitates the exchange of learning objects among organizations and individuals.

The term "learning object" is not intended to be restrictive but refers to any digital asset that can be used to enable teaching or learning (IEEE, 2001). A learning 
object does not imply some specific size or modularity. It may refer to many different types of objects from simple images or video clips, through complex questions, to collections of objects arranged in one or more sequences. One critical issue about learning objects concerns the ability of their administration, in terms of updating, identifying, utilizing, sharing, and reusing them, which remains a great challenge, as their number continues to grow at a fast rate. The only viable solution proposed to this problem is to define a set of metadata on them, that is, a set of attributes required to fully and adequately describe them (IEEE, 2001). There are several, highly active, standardization initiatives today that are concerned with the definition of specifications for learning resources metadata.

The LRBS usually offer learning objects stored in digital repositories. While digital repositories, in the broadest sense, are used to store any digital material, digital repositories for learning objects are considerably more complex, both in terms of what needs to be stored and of how it may be delivered (Duncan, 2002). Digital repositories are not mere portals, i.e., gates of access to learning material. What makes a digital repository much more than a portal is the ability to discover a learning object and put it to a new use. The purpose of a digital repository is not simply safe storage and delivery but also reuse and sharing. In a few cases, LRBS contain digital repositories, but this is not always the case.

An important aspect of LRBS is the categories of users that benefit from them, by performing certain usage scenarios. Users of digital repositories are mostly educators and, in general, authors of learning content. They may produce Webbased courses or classroom courses, face-to-face or distance learning, or full courses or short digital "nuggets." The LRBS should be neutral to the pedagogic purposes of the material, just as a library has no influence over where or when a book is read.

One can mention a lot of e-learning resources repositories. Unfortunately, the various repositories are either closed systems or systems that allow user access only through proprietary interfaces and data formats. In brief, there is lack of interoperability. Interoperability can be defined (IEEE, 1990) as "the ability of two or more systems or components to exchange information and to use the information that has been exchanged." To a user, the lack of interoperability means the following:

- Applications and their data are isolated from one another.

- Redundant data entry is common.

On the contrary, interoperability

- Ensures that data are entered only once in one application and automatically propagates to other applications 
- Allows applications to exchange data more effectively

- Defines the rules of interaction among software applications

The aim of this chapter is to present the requirements of an ideal e-learning resources repository that will provide services for covering the aforementioned critical issues. We will also describe how this system could be noncentralized, which is the main difference from the system that exists today in the World Wide Web (WWW). Peer-to-peer (P2P) based approaches are more flexible than centralized approaches and have several advantages. For example, imagine that content consumers, both teachers and students, will benefit from having access not only to a local repository, but to a whole network, using queries over the metadata of learning objects that will be distributed (Nejdl et al., 2002).

The structure of this chapter is as follows. We start by analyzing and comparing the functionalities of various e-learning resources repositories under evaluation. This analysis and comparison lead to the extraction of the tasks and the requirements that an ideal e-learning resources repository should support. We continue by focusing on the special features that an ideal system should present. The special features will be illustrated by using case diagrams and scenarios in order to make them more clear to the reader. In the sequence, we will describe architecture for interoperable repositories. Apart from a central repository where the user can find learning resources, several other repositories located in different places in the Internet can be accessed in order to allow the user to perform a request for specific-learning resources at a network of repositories. The communication among the repositories can be performed via designated interfaces, which can import and export the metadata of their learning resources. The exchange of the metadata can be accomplished through a descriptive and extensive language such as XML.

\section{E-Learning Resources Brokerage System}

In this section, we focus on the requirements that an e-Learning Objects Brokerage System must satisfy, after having examined several e-learning objects brokerage systems. The requirements are grouped in tasks that the system has to perform. The type of task analysis we have chosen is hierarchical and borrows ideas from several sources, including Wigley (1985). In a hierarchical task analysis, according to Stammers et al. (1990), each task is analyzed by 
"breaking it into task elements or goals which become increasingly detailed as the hierarchy progresses." The most general information is placed at the top of the hierarchy, with the more specific information following on lower levels.

Currently, there are several e-learning objects brokerage systems operating on the WWW. Each offers certain functionalities, such as browsing and searching in a catalog of resources, managing an e-portfolio of favorite resources, booking resources, annotating resources, contributing resources, etc. Typical examples of such systems are as follows:

- $\quad \operatorname{SeSDL}$ (http://www.sesdl.scotcit.ac.uk)

- LearnAlberta Portal (http://www.learnalberta.ca/)

- CAREO (http://careo.netera.ca)

- COLIS (http://www.edna.edu.au/go/browse/0)

- $\quad$ SMETE (http://www.smete.org/)

- $\quad$ MERLOT (http://www.merlot.org)

- Heal (http://www.healcentral.org/index.htm)

- Universal Brokerage Platform for Learning Resources (http:// www.educanext.org)

- European Knowledge Pool System (http://rubens.cs.kuleuven.ac.be:8989/ lkptm5/intro.jsp)

- World Lecture Hall (http://www.utexas.edu/world/lecture/)

- Globewide Network Academy (http://www.gnacademy.org/)

- $\quad$ Element K (http://www.elementk.com/)

- Online Learning Network (http://www.onlinelearning.net/)

- DigitalThink (http://www.digitalthink.com/)

- McGraw-Hill Learning Network (MHLN) (http://www.mhln.com/)

- IntraLibrary (http://www.intrallect.com/)

Table 1 summarizes the functionality of all the LRBS that have been examined and gives a comparative view. In Table 1, if a system performs a certain task, it is given a value of 1 ; otherwise, it is given a value of 0 . In the same table, there is a column that illustrates the percentage of systems that perform each task.

Some immediate and useful remarks can be drawn from Table 1. First, almost all the general tasks appear in most LRBS in the sample set. Some general tasks, such as "contribute resource," appear to have a lower percentage. This can be easily explained if we consider that some of the systems in the survey's set are 
Retalis

Table 1: Comparing Brokerage System Tasks

\begin{tabular}{|c|c|c|c|c|c|c|c|c|c|c|c|c|c|c|c|c|}
\hline \multirow[t]{2}{*}{ TASKS } & \multirow{2}{*}{ STATS $(\%)$} & \multicolumn{15}{|c|}{ PLATFORMS } \\
\hline & & UBP & WLH & GNA & EL.K & $\mathrm{OLn}$ & Dig.Th & $\mathrm{McGr}$ & SeSDL & IntL & Heal & Colis & s Careo & Merlot & Smete & Learn A \\
\hline Browse catalog of learning objects & 93 & 1 & 1 & 1 & 1 & 1 & 1 & 1 & 1 & 1 & 0 & 1 & 1 & 1 & 1 & 1 \\
\hline View catalog of learning objects & 80 & 1 & 0 & 1 & 1 & 1 & 1 & 1 & 1 & 1 & 0 & 1 & 1 & 1 & 1 & 0 \\
\hline Browse learning objects by area/category & 93 & 1 & 1 & 1 & 1 & 1 & 1 & 1 & 1 & 1 & 0 & 1 & 1 & 1 & 1 & 1 \\
\hline Search learning objects & 93 & 1 & 1 & 1 & 1 & 1 & 0 & 1 & 1 & 1 & 1 & 1 & 1 & 1 & 1 & 1 \\
\hline Simple text search & 93 & 1 & 1 & 1 & 1 & 1 & 0 & 1 & 1 & 1 & 1 & 1 & 1 & 1 & 1 & 1 \\
\hline Advanced search & 80 & 1 & 1 & 0 & 1 & 0 & 0 & 1 & 1 & 1 & 1 & 1 & 1 & 1 & 1 & 1 \\
\hline Customized query search & 7 & 1 & 0 & 0 & 0 & 0 & 0 & 0 & 0 & 0 & 0 & 0 & 0 & 0 & 0 & 0 \\
\hline Sort results & 47 & 1 & 1 & 0 & 0 & 0 & 0 & 1 & 1 & 1 & 0 & 0 & 0 & 1 & 1 & 0 \\
\hline View learning object details & 100 & 1 & 1 & 1 & 1 & 1 & 1 & 1 & 1 & 1 & 1 & 1 & 1 & 1 & 1 & 1 \\
\hline View learning object metadata & 100 & 1 & 1 & 1 & 1 & 1 & 1 & 1 & 1 & 1 & 1 & 1 & 1 & 1 & 1 & 1 \\
\hline View comments, reviews, and ratings & 20 & 0 & 0 & 0 & 0 & 0 & 0 & 0 & 0 & 0 & 0 & 0 & 1 & 1 & 1 & 0 \\
\hline View cross-referenced learning objects & 13 & 0 & 0 & 0 & 0 & 1 & 0 & 0 & 0 & 0 & 0 & 0 & 0 & 0 & 1 & 0 \\
\hline Reserve learning object & 47 & 1 & 0 & 0 & 1 & 0 & 0 & 0 & 1 & 1 & 1 & 0 & 1 & 0 & 0 & 1 \\
\hline Agree with license agreement & 13 & 1 & 0 & 0 & 0 & 0 & 0 & 0 & 0 & 0 & 1 & 0 & 0 & 0 & 0 & 0 \\
\hline Book learning object & 33 & 1 & 0 & 0 & 0 & 0 & 0 & 0 & 1 & 1 & 1 & 0 & 1 & 0 & 0 & 0 \\
\hline Add to shopping cart & 13 & 0 & 0 & 0 & 1 & 0 & 0 & 0 & 0 & 0 & 0 & 0 & 0 & 0 & 0 & 1 \\
\hline Manage reserved learning objects & 67 & 1 & 1 & 0 & 1 & 0 & 0 & 0 & 1 & 1 & 1 & 0 & 1 & 1 & 1 & 1 \\
\hline View list of booked learning objects & 33 & 1 & 0 & 0 & 0 & 0 & 0 & 0 & 1 & 1 & 1 & 0 & 1 & 0 & 0 & 0 \\
\hline View shopping cart & 13 & 0 & 0 & 0 & 1 & 0 & 0 & 0 & 0 & 0 & 0 & 0 & 0 & 0 & 0 & 1 \\
\hline Commit reservation & 27 & 0 & 0 & 0 & 0 & 0 & 0 & 0 & 1 & 1 & 1 & 0 & 0 & 0 & 0 & 1 \\
\hline View history of all reserved learning objects & 40 & 1 & 0 & 0 & 1 & 0 & 0 & 0 & 1 & 1 & 0 & 0 & 1 & 0 & 0 & 1 \\
\hline Categorize learning objects (e.g., favorites) & 0 & 0 & 0 & 0 & 0 & 0 & 0 & 0 & 0 & 0 & 0 & 0 & 0 & 0 & 0 & 0 \\
\hline Comment, review, or rate a learning object & 20 & 0 & 1 & 0 & 0 & 0 & 0 & 0 & 0 & 0 & 0 & 0 & 0 & 1 & 1 & 0 \\
\hline Buy learning object (payment) & 27 & 0 & 0 & 0 & 1 & 1 & 1 & 0 & 0 & 0 & 0 & 0 & 0 & 0 & 0 & 1 \\
\hline Learning object delivery & 100 & 1 & 1 & 1 & 1 & 1 & 1 & 1 & 1 & 1 & 1 & 1 & 1 & 1 & 1 & 1 \\
\hline Connect to system server & 60 & 1 & 0 & 0 & 1 & 0 & 1 & 1 & 1 & 1 & 1 & 0 & 1 & 0 & 1 & 0 \\
\hline Connect to another site (provider) & 60 & 1 & 1 & 1 & 1 & 1 & 0 & 0 & 0 & 0 & 0 & 1 & 1 & 1 & 1 & 0 \\
\hline Send to customer (via mail) & 13 & 0 & 0 & 0 & 1 & 0 & 0 & 0 & 0 & 0 & 0 & 0 & 0 & 0 & 0 & 1 \\
\hline
\end{tabular}

Copyright (C) 2005, Idea Group Inc. Copying or distributing in print or electronic forms without written permission of Idea Group Inc. is prohibited. 


\section{Table 1: Comparing Brokerage System Tasks (continued)}

\begin{tabular}{|c|c|c|c|c|c|c|c|c|c|c|c|c|c|c|c|c|}
\hline \multirow[t]{2}{*}{ TASKS } & \multirow{2}{*}{ STATS $(\%)$} & \multicolumn{15}{|c|}{ PLATFORMS } \\
\hline & & UBP & WLH & GNA & EL.K & OLn & Dig.Th| & $\mathrm{McGr}$ & SeSDL & LIntL & Heal & Colis $\mathrm{C}$ & Careo & Merlot & Smete & LearnA \\
\hline Contribute learning object & 60 & 1 & 1 & 1 & 0 & 0 & 0 & 1 & 1 & 1 & 0 & 1 & 1 & 1 & 0 & 0 \\
\hline Upload to system server & 27 & 1 & 0 & 0 & 0 & 0 & 0 & 1 & 1 & 1 & 0 & 0 & 0 & 0 & 0 & 0 \\
\hline Provide link to another site & 47 & 1 & 1 & 1 & 0 & 0 & 0 & 1 & 0 & 0 & 0 & 1 & 1 & 1 & 0 & 0 \\
\hline Define terms (license agreement) & 20 & 1 & 0 & 0 & 0 & 0 & 0 & 0 & 0 & 0 & 0 & 0 & 1 & 1 & 0 & 0 \\
\hline Manage contributed learning objects & 47 & 1 & 0 & 1 & 0 & 0 & 0 & 1 & 1 & 1 & 0 & 0 & 1 & 1 & 0 & 0 \\
\hline View list of contributed learning objects & 40 & 1 & 0 & 0 & 0 & 0 & 0 & 1 & 1 & 1 & 0 & 0 & 1 & 1 & 0 & 0 \\
\hline Edit/cancel contributed learning object & 47 & 1 & 0 & 1 & 0 & 0 & 0 & 1 & 1 & 1 & 0 & 0 & 1 & 1 & 0 & 0 \\
\hline Commit contribution (make available) & 27 & 0 & 0 & 0 & 0 & 0 & 0 & 0 & 1 & 1 & 0 & 0 & 1 & 1 & 0 & 0 \\
\hline Personal user account & 80 & 1 & 0 & 0 & 1 & 1 & 1 & 1 & 1 & 1 & 1 & 0 & 1 & 1 & 1 & 1 \\
\hline User profile and preferences & 80 & 1 & 0 & 0 & 1 & 1 & 1 & 1 & 1 & 1 & 1 & 0 & 1 & 1 & 1 & 1 \\
\hline My library/portofolio of learning objects & 60 & 1 & 0 & 0 & 1 & 1 & 0 & 1 & 1 & 1 & 1 & 0 & 1 & 1 & 0 & 0 \\
\hline Site personalization & 7 & 0 & 0 & 0 & 0 & 1 & 0 & 0 & 0 & 0 & 0 & 0 & 0 & 0 & 0 & 0 \\
\hline Update notification & 80 & 1 & 0 & 1 & 1 & 1 & 1 & 1 & 0 & 0 & 1 & 1 & 1 & 1 & 1 & 1 \\
\hline Mailing list & 20 & 0 & 0 & 0 & 0 & 1 & 1 & 0 & 0 & 0 & 1 & 0 & 0 & 0 & 0 & 0 \\
\hline Newsletter & 33 & 0 & 0 & 0 & 1 & 0 & 1 & 1 & 0 & 0 & 0 & 1 & 0 & 0 & 0 & 1 \\
\hline What's new/upcoming updates & 60 & 1 & 0 & 1 & 0 & 0 & 0 & 1 & 0 & 0 & 1 & 1 & 1 & 1 & 1 & 1 \\
\hline System informative material & 100 & 1 & 1 & 1 & 1 & 1 & 1 & 1 & 1 & 1 & 1 & 1 & 1 & 1 & 1 & 1 \\
\hline Help manual & 73 & 1 & 0 & 0 & 0 & 0 & 1 & 1 & 1 & 1 & 1 & 1 & 1 & 1 & 1 & 1 \\
\hline F.A.Q. & 73 & 1 & 1 & 1 & 1 & 1 & 1 & 1 & 0 & 0 & 0 & 1 & 1 & 1 & 0 & 1 \\
\hline Site map & 53 & 0 & 1 & 0 & 1 & 1 & 1 & 0 & 0 & 0 & 1 & 1 & 0 & 1 & 0 & 1 \\
\hline Terms of use & 73 & 1 & 0 & 1 & 1 & 1 & 1 & 1 & 1 & 1 & 0 & 1 & 0 & 0 & 1 & 1 \\
\hline Glossary (of technical terms) & 20 & 0 & 0 & 0 & 0 & 0 & 0 & 0 & 0 & 0 & 0 & 1 & 1 & 0 & 0 & 1 \\
\hline Company informative material & 100 & 1 & 1 & 1 & 1 & 1 & 1 & 1 & 1 & 1 & 1 & 1 & 1 & 1 & 1 & 1 \\
\hline Company profile (about us) & 100 & 1 & 1 & 1 & 1 & 1 & 1 & 1 & 1 & 1 & 1 & 1 & 1 & 1 & 1 & 1 \\
\hline Partners and alliances & 87 & 1 & 0 & 0 & 1 & 1 & 1 & 1 & 1 & 1 & 1 & 1 & 1 & 1 & 1 & 1 \\
\hline News and events/calendar & 67 & 0 & 0 & 1 & 1 & 1 & 1 & 0 & 1 & 1 & 0 & 1 & 0 & 1 & 1 & 1 \\
\hline Contact system personnel & 100 & 1 & 1 & 1 & 1 & 1 & 1 & 1 & 1 & 1 & 1 & 1 & 1 & 1 & 1 & 1 \\
\hline E-mail (contact us) & 100 & 1 & 1 & 1 & 1 & 1 & 1 & 1 & 1 & 1 & 1 & 1 & 1 & 1 & 1 & 1 \\
\hline
\end{tabular}

Copyright (C) 2005, Idea Group Inc. Copying or distributing in print or electronic forms without written permission of Idea Group Inc. is prohibited. 
Table 1: Comparing Brokerage System Tasks (continued)

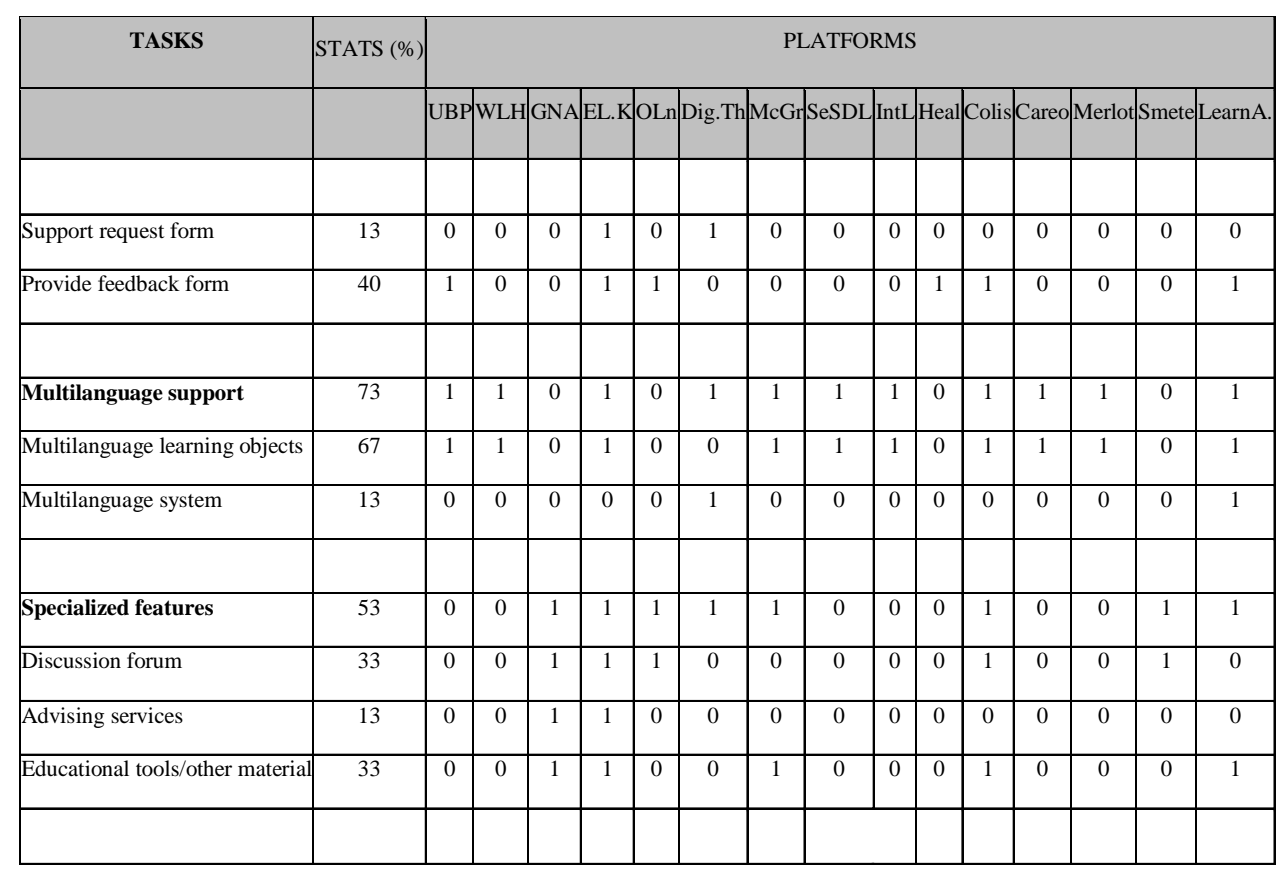

actually "providers" of e-learning content and not open "brokers," and thus, they do not support contribution of user material.

Regarding "browsing," there is nothing much to be said, because, as expected, almost all systems support this feature. Regarding the issue of searching the learning content, almost all systems provide some sort of simple text search. However, only about three out of four of the systems provide an option for advanced search and sorting of the results, and even worse, only a small percentage allows for actual customized query-based search. Although "viewing a resource's details" is also implemented by all systems, this feature is limited to viewing a resource's metadata. Only few systems offer "previewing" of the material or an adequate summary. Comments and ratings from other users and cross-referenced resources are also absent from most systems.

As Table 1 indicates, about half of the systems support "reservation of resources." The user is therefore forced to commit to his or her choice and proceed to the resource delivery or payment, without having the option of collectively reviewing his or her choices. Systems that have implemented the 
resource-reserving feature provide only a limited functionality on managing the reserved resources, by providing an option to view the reserved resources and cancel a reservation. No system provides functionality about viewing all the reserved resources (and not just those of the last transaction), annotating them and categorizing them.

We can, also, observe that some systems that sell e-learning content do not support a very critical feature in the selling process, namely, the "online payment" feature. This should be considered as a drawback for such systems, because it forces the user to interrupt a process and get involved in a separate process in order to achieve his or her goal. "Resource delivery" is implemented by all systems, because this is the ultimate goal of an LRBS. The delivery of the resource can be either by downloading from the system server or by connecting to some external site, depending on the system's architecture and goals. It is also possible that some material may be delivered via mail to the customer.

"Contribution of resource" is a feature that clearly does not refer to all LRBS. But even systems that allow the contribution of resources usually do so partially, because most of them do not allow the user to specify the conditions under which the resource is distributed or do not allow the removal of a contributed resource. Again, the user is forced to commit early to his or her choice. It should be possible for the user to contribute a resource and keep it private, until the user decides to offer it openly.

Although a significant percentage of the systems provide personal user accounts, most of them do not utilize this beyond some basic level. Only few systems allow for personalization based on the users' preferences. LRBS update their content often and should therefore provide some mechanism for notifying their users. Some systems do not comply with this requirement, while others do so in more than one way.

All the systems provide "help" in more than one form, predominantly, the FAQ form. It is, however, surprising that only about three out of four of the systems provide an actual system manual, and that only one out of four systems provide a glossary of technical terms that may be abundant in LRBS. All systems provide an e-mail address so to the user can contact the system's personnel for support or feedback. However, only a small percentage provides more sophisticated and structured ways to submit a support request or provide feedback.

An interesting point is that although nearly three out of four of the systems allow and properly support multilingual content, only a small percentage of the systems account for multilingual support within the system itself. Finally, we see that more than half of the systems provide additional specialized features of some sort, with the ones most popular being the option for discussion forums and educational tools. 


\section{Functionality and Services}

When examining the functionality and the services offered by the brokers, one can create a superset of these functions and form the ideal functionality. This superset is presented in this section and can be considered the requirements specifications for an "ideal" e-learning objects brokerage system.

The major tasks that LRBS perform are as follows:

1. Browse catalog of resources

2. Search resources

3. View resource details

4. Reserve details

5. Manage reserved resource

6. Buy resource (payment)

7. Deliver resource

8. Contribute resource

9. Manage contributed resources

10. Annotate resource

11. Offer personal user account

12. Update notification

13. Provide system informative material

14. Provide company informative material

15. Contact system personnel

16. Offer multilanguage support

17. Offer specialized features

It is evident that every system should provide some way of browsing and searching for the offered resources. It is cleared that a simple text search is not sufficient, and some sorting of the search results should be available. Therefore, we propose that an ideal e-learning objects brokerage system implements the following two general tasks: "browse catalog of resources" and "search resources." Browsing should concern all resources on a specific (easily selected) area/category. As for searching, in addition to the simple text search, an advanced and customized search option should be available. The results should be presented, after being sorted, either alphabetically, by relevance, by category, by last update, or by any other metadata information available for the resources. 
When viewing the details of a selected resource, it is useful for the user to view, in addition to the metadata available for the resource, some other indicative information. This includes some sample material or a summary/abstract of the resource, depending on each case. Users also seem to find comments and ratings by other users that have used the same resource to be useful. The e-learning objects brokerage system should also offer cross-references to other resources that were also used by users of a given resource. This seems to provide the user with a very focused and high relevancy search option, as illustrated by sites like "Amazon" and "Google" (with the option "Find similar pages").

In the case that an e-learning objects brokerage system requires some form of resource reservation (as in brokerage platforms or providers of e-learning content), the system should provide the user with the option to view the "license agreement" under which the reservation (or buying) of resources takes place, at any time (before, during, or after the reservation takes place). The "license agreement" can be either specific to each resource (as in brokerage platforms, where resources have different providers) or common to all resources (as in providers of e-learning content, where the provider offers all resources). The user should have the "Reserve resource" option available, without being forced to commit to his or her choice, until the user is ready to proceed to the next step (resource delivery or payment).

Except for reserving a resource, the user should also be able to somehow manage the reserved resources. This option is not limited to viewing the resources reserved during the user's last transaction but may (preferably) include all the reservations (that were actually committed) by the user in the past. This allows the user to manipulate this list by designating his or her favorite resources, recommend a resource for other users, rate a resource, and comment (on usefulness, relevance to some topic, or any other useful criterion). The user can also categorize the resources to custom categories and manage the resources (actually links to the resources). This includes canceling an already reserved resource or committing to the reservation (at which time the resource's provider should be notified, and not prior to that time).

The option to buy a resource is critical in LRBS that "sell" e-learning content online. Although the payment stage of a transaction can be carried out via alternative offline methods (e.g., telephone or mail order), we feel that because the rest of the transaction is completed online, so must the payment stage. The subtasks for implementing this requirement are well known and need not be discussed here. We should note, however, that the payment stage should be in accordance with the reservation of resources and the commitment requirement as explained above. Hence, the user should be allowed to reserve and cancel the reservation for any number of resources before committing and paying for them. 
Regarding the delivery of resources, this can be implemented depending on the resource type, system category, terms of resource sharing (e.g., use once, unlimited use), and its digital rights, in general. This could include presenting the e-learning material onscreen, downloading the material to a local media, or linking to a Web site. In case an e-learning brokerage system contains a digital repository, it will be able to provide access to the e-learning content by itself. In any other case, it should provide only access details that should have already been given by the content provider as an addition to the standard learning object metadata.

Complementary to the resource delivery is the option to contribute a resource. This is not required by all LRBS, but it is necessary for digital repositories. When contributing a resource, the user should be able to either provide a link to the resource or upload the material to the system server, according to the desired functionality of the system. In any case, the user should be able to clearly define the intended viewers of the resource and the conditions under which the resource may be used, i.e., the digital rights. The system is responsible to uphold any constraints defined on the resources, provided that these comply with the system's policy.

An assistant functionality to contributing a resource is the "Manage contributed resources" feature. In addition to viewing the resources contributed by a userprovider, the user should have the option to edit a contributed resource or even cancel a contribution and withdraw the resource, again given that this complies with the system's policy. Last, the user has the option to make a contribution public and thus commit to his or her contribution.

The user should be provided with an option to annotate a resource and store the annotations in an annotation repository. The user should be able to comment on the resource, using either free text or specific notations, e.g., "star system" for rating the quality of the resource. There should be an authentication mechanism for each user, because there can be two kinds of annotations: the private ones and the public ones. Each annotation object should be accompanied by metadata specifying the author, a time stamp, the kind (e.g., "criticism," "praise," etc.). Additionally, other relevant subtasks are to filter and retrieve annotation sets based on their metadata.

The option to create a personal user account is almost a necessity in e-learning objects brokerage systems. This allows the system to keep personal user information (e.g., the reserved resources), to contact the user for updates, and to adjust to each user's individual needs. The latter is important in order to provide a personalized and thus efficient and focused use of the system, because each user has unique expectations from the system.

Regarding the "Update notification" option, this should be provided upon the user's request only, and the user should be able to terminate it at any time. The 
information provided should be relevant to the user as possible, something that can be achieved by utilizing the user's personal preferences. The notification should be made both online (e.g., in the home page or some specific news page) and via e-mail (e.g., mailing list or newsletter), according to the user's request. An important feature of any system is to provide informative material about the system. This material can and should take many different forms, including manual, FAQ, site map, and glossary. The user should have the option to select the form with which he or she feels most comfortable with and believes it can most efficiently and accurately provide the needed information. It is also important that the information be presented modularly, starting from help on the basic system functionality and moving to the more advanced functionality upon user request. Lists of steps that guide the user should be used whenever possible, instead of plain text.

The systems should also provide company informative material that although not directly related to the system itself, may provide useful information to some users. This information should be clearly marked and accessible but should not interfere with the system's functionality and documentation. The latter will result in confusing the user and blurring the system's intended goals and capabilities.

Besides reading precompiled help material, the system should also provide an option to contact the system personnel. The user should have the option to contact (via e-mail, phone, or online live chat, according to the importance of the request) the system personnel and get answers to specific questions or provide feedback about the system. Support and feedback should be preferably implemented via form completion. The structured input guides the user and allows for better processing of information.

The multilanguage support feature should be considered among the most important features of an LRBS. A system that provides e-learning content should be able to also address the needs of foreign users that may not master the language of the system. This, of course, is not limited to providing multilanguage resources, which is equally important. The entire system documentation and online information (except contributed resources) should be able to be translated to other languages. A clearly marked way should be provided to toggle between languages, appearing (preferably) on the home page (or every page) through icons (e.g., country flags).

The above cover the basic requirements of LRBS. In addition, some specialized features may also be present, depending on the system's goals. Such features include discussion forums, glossaries, etc. Although these features are not considered to be essential, when implemented and integrated correctly, they can advance a system's overall image. 


\section{Designing an Ideal Decentralized System}

Most of the existing LRBS are based on a centralized, nondistributed architecture. All the offered learning resources can be found in a central repository of data to which the broker has access. The research and development challenge is to build systems with architectures of distributed data repositories. Apart from a central data repository, where the broker can find its own learning resources, several other data repositories located in different places on the Internet can connect to such a decentralized brokerage system. In particular, each e-learning resources brokerage system or any other independent digital repository can register to this brokerage system. Whenever a user performs a request to the broker for specific learning resources, the broker will search in its digital repository and communicate with the external brokerage systems or digital repositories. The communication with the other systems can be performed via designated interfaces, which can import and export the metadata of their learning resources. The exchange of metadata can be accomplished through a descriptive and extensive language such as XML. Importing the XML representation of metadata, the broker can be informed about the kinds of learning resources that other systems possess. Figure 1 illustrates an overview of the design of a decentralized e-learning objects brokerage system.

Another additional functionality that LRBS should support is the synchronization of the metadata descriptions of their learning resources. A synchronization process means that a LRBS could decide to provide a replicate of the metadata descriptions of their learning resources to another system, e.g., for

Figure 1: Overview of the design of a decentralized e-learning brokerage system

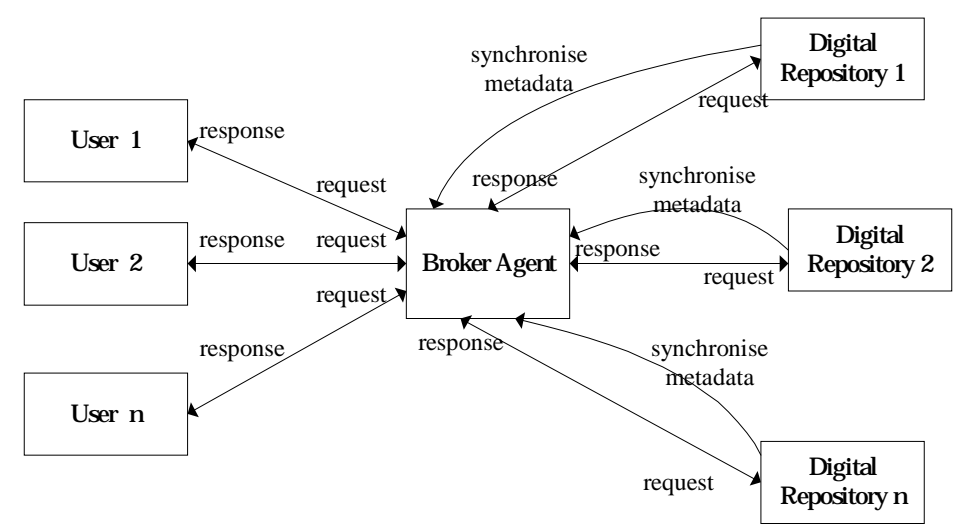

Copyright (C) 2005, Idea Group Inc. Copying or distributing in print or electronic forms without written permission of Idea Group Inc. is prohibited. 
wider dissemination of their resources. In this case, each alteration, creation, and deletion of the metadata description of a learning resource could appear in more than one LRBS. The LRBS will collaborate in order to perform an update or an insert or delete command at their remote metadata repositories.

Following this design principle, a brokerage system can be characterized from an open and interoperable architecture, where various and different delivery systems and repositories that offer learning resources can communicate. The basic prerequisite for enabling interoperability is that each digital repository should fully support the same metadata standard (e.g., IMS LOM, IEEE LOM, etc.).

\section{System Implementation}

In order for the above communication to be established, a specific interface for each digital repository must be developed. Each interface is being implemented as a "Java Web Service" and is responsible for the achievement of the

Figure 2: System architecture and application flow

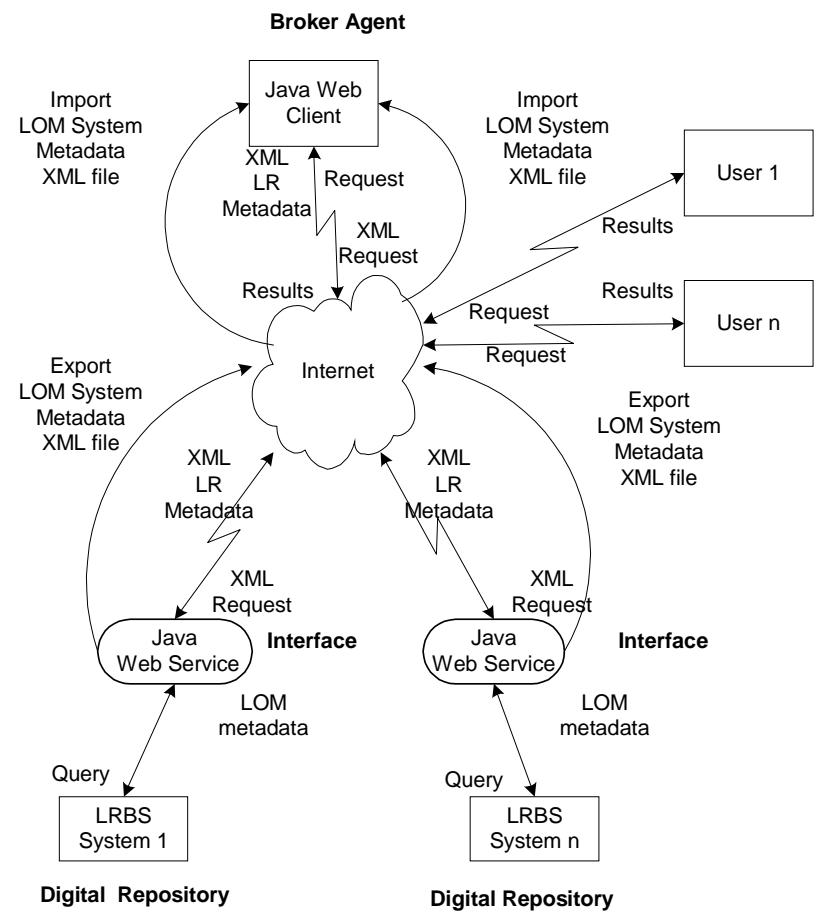

Copyright (C) 2005, Idea Group Inc. Copying or distributing in print or electronic forms without written permission of Idea Group Inc. is prohibited. 
communication between the repository and the broker. This communication will be based on the interchange of metadata files. The broker-agent will compose a Java Web client that will communicate with each Java Web service. Figure 2 illustrates the architecture of the described system, presenting the information flow inside the application.

The first thing that has to be done is the registration process. Through that process, each digital repository registers to our system. The administrator of the repository has to define the information that the search engine needs in order to communicate with the repository. The information contains the IP address or host name of its interface and the port number in which the interface will listen for queries from the search engine. In a future version of the implementation work, the interface will also be able to export the taxonomy of its metadata structure. The search engine will register the repository and provide "guidance" on the communication protocol. Particularly, the search engine will give the method name that each interface must implement in order to be able to provide the requested metadata. It will also give the way it will call that method and the arguments that needed to be passed through the call. That method will be standard for all the interfaces that want to communicate with our system.

The application flow starts from the time a user wants to search for learning resources (Request). After the user enters the selection criteria, the broker agent (or search engine) calls the interface of each digital repository (through the given IP address and port number) and passes, through the predefined method, the user request/query through an XML file (XML Request).

The interface of the LRBS interacts with its LOM subsystem when passing its query (Query). The LOM subsystem responds to the interface returning the LOM Metadata that satisfy the query (LOM Metadata). Once the interface has the requested metadata, it transforms the metadata into an XML format and returns them to the broker agent (XML LR Metadata). Eventually, the broker agent returns the metadata on the user's screen in a readable format (Results). Each one of the LRBS has an interface, which is implemented as a Web service. The interface implementation is based on the LOM System and is independent from the search engine's implementation. The only requirement in order for the search engine-interface communication to be established is the existence of a method that is called "getLRMetadata(XMLQuery)". The method gets as an argument an XML file that contains the query of the metadata that the user requests and returns to the search engine an XML file that contains the LR Metadata that the LOM System returns to its interface/Web service. Figure 3 illustrates a sequence diagram that describes the exchange of the metadata. 
Figure 3: Metadata exchange

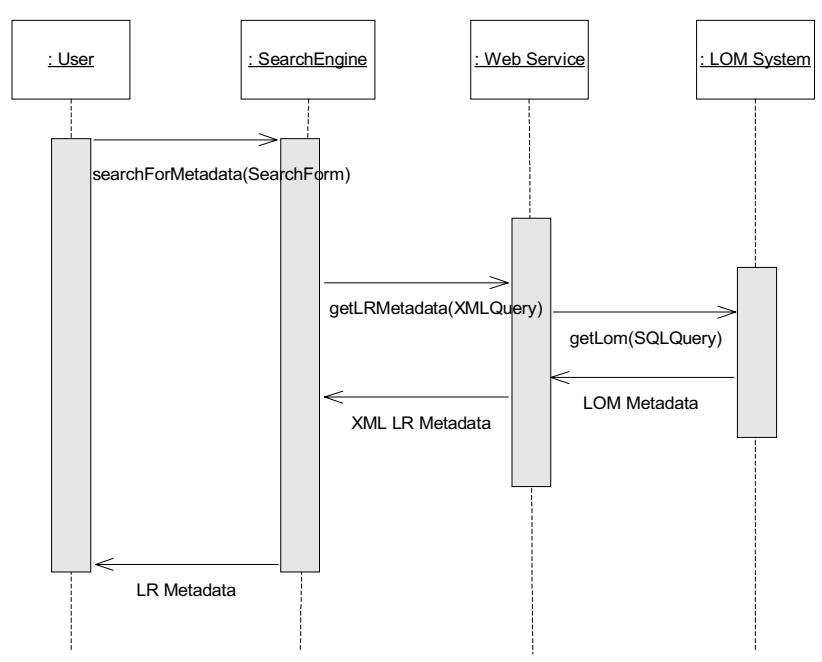

\section{Discussion}

The idea of interoperable LRBS is becoming popular. Several groups have started experimenting and standardizing the interoperability process. The IMS Digital Repositories Interoperability (DRI) specification aims to provide recommendations for the interoperation of the most common repository functions. The ultimate aim is to make recommendations that could be turned to implementable services via common interfaces (IMS, 2001). DRI defines a general reference model that captures all instances of possible implementations, such as the following:

- A user searching a repository directly

- A user conducting a search across repositories via a Search Gateway intermediary (acting as a translator)

- A user conducting a search across repositories via a Harvest intermediary (acting as an aggregator)

At technical level Z39.50 (http://lcweb.loc.gov/z3950/agency/), which is widely used for searching at digital libraries, a searcher is permitted to use the familiar user interface of the local system to search the local library catalog as well as any remote database system that supports the standard. While Z39.50 is assumed to be used for searching systems such as digital libraries, XQuery is 
recommended as the preferred query mechanism for XML-based learning object repositories.

Another group that is working on a test bed for a network of distributed repositories using SOAP-based messaging is the Learning Objects Network, Inc. (http://www.learningobjectsnetwork.com/). Learning Objects Network, Inc. (LON) demonstrated a working model using messaging and metadata search capabilities like that recommended in the IMS DRI specifications at the January 2002 IMS meetings in Cambridge, MA.

Furthermore, The OpenURL is a framework for an open and context-sensitive method of reference linking that is gaining widespread acceptance in the publishing and library communities. Rather than seeking to be independent of physical location, the advantage of OpenURL resolution is finding the appropriate copy or copies of an item that are stored in multiple locations (see http:// www.sfxit.com/openurl/openurl.html for more information). Although OpenURL has been developed in the context of scholarly literature, a framework for generalizing the model to other domains has been put forward (the 'Bison-Fute' model—see http://www.dlib.org/dlib/july01/vandesompel/07vandesompel.html). This generalized model could be used as the basis for adoption within the IMS DRI community.

On the JISC-funded OLIVE project, there is ongoing research and development in the distributed querying of learning object repositories. Basically allowing LMS and, in their case, OpenURL resolves to find and retrieve learning objects such as online courses. OpenURL has been fast-tracked by NISO for adoption as a NISO standard (http://www.niso.org/).

Recognizing the fact that learning objects are still a new concept as well as the reusability, exchange, and interoperability of learning resources are significant issues, we have to think of possible obstacles that delay the R\&D achievements. These obstacles are as follows:

1. The lack of consensus about the definition and description of learning objects as well as their granularity. Perceptions about the nature and size of learning objects differ. One could easily find out that the main learning objects repositories do not conform to the LOM standards. For example, while IntraLibrary (http://www.intrallect.com/) and Merlot (http:// www.merlot.org/) are IMS compliant, Belle/Careo (http://careo.netera.ca/) is using the CanCore protocol, which is a simplification and interpretation of the 86 elements of the IMS Learning Resource Metadata Information Model. Moreover, Colis (EdNA) (http://www.edna.edu.au/go/browse/0/) depends on the EdNA Metadata Standard, which is based on Dublin Core Metadata Element Set. Other R\&D groups have proposed quite different sets of metadata (in the best case, some of them are extended versions of the IMS standard) in order to describe Web-based multimedia teaching 
materials in a specific domain. For example, Heal (http:// www.healcentral.org/) has developed a standard metadata specification for sharing medical education multimedia based on the IMS standard. Other ideas come around, like the ones proposed in the UNIVERSAL project (http://www.educanext.org): "LOM does not propose learning resource types, which would be required for categorizing educational activities. At the Universal Brokering Platform, the following educational activity types are introduced: case study, course, course unit, exam, exercise, experiment, group work, lecture, presentation, and project." Furthermore, despite the fact that sites like Math Goodies, which is a free math help site featuring interactive lessons, homework help, worksheets, etc., do not use LOM description, they are very popular. Users prefer resources like lesson plans that do not entirely fit into a LO category.

2. The lack of clarity on how to reuse learning objects and create new learning resources. It is evident that learning objects cannot work like Lego. On the one hand, we could affirm that instructional design methods, which could effectively support the process of aggregating course content, do not exist. In fact, there are some ideas similar to that presented by Douglas (2001) that propose a component-based instructional development process, and Douglas argues that we should adopt/adapt object-oriented software design methods. On the other hand, authoring tools and learning content management systems (or even learning management systems) are not advanced enough to create content "on-the-fly" from learning objects. Very few commercial products of this type exist. One prominent example of such a tool could be the Designer's Edge (http://www.allencomm.com/ products/authoring_design/designer/). The unavailability of usable tools is surprising, because research efforts have only started with the European Union DELTA program [e.g., DIScourse project (http://www.itd.ge.cnr.it/ sarti/papers/mispelkampsarti.html)]. The reusability of LOs is still a tacit knowledge.

3. The insufficient description of the "behavior" of learning objects. Despite the fact that there are many attributes in learning object metadata description, they do not fully capture the "behavior" of a learning object. A learning object is created with specific learning objectives in mind, holds specific behavior, and interoperates with surrounding learning objects. Isolating a learning object and reusing it means that either this learning object can remain intact, because it might fit well to the new learning context, or this learning object needs changes. In the latter and most usual case, not only do technological problems arise but also instructional. A learning object does not only have its own characteristics and learning value, but its relationship with other learning objects offers additional learning experiences. Descriptive models such as CLEO or educational 
modeling languages such as EML have been suggested. However, we should also design models for the authoring/aggregation of learning content. We need to adapt formal design models and methods from the field of hypermedia engineering (e.g., OOHDM, RMM, etc.). Such models will show which learning object consists of a learning application and how these learning objects are interrelated. Of course, these models as well as their formal notations (and bindings) should be compatible with the existing (or the ones that might arise) learning technology standards like the Content Packaging, Learning Design, etc. One approach akin to a modeling notation in education is concept mapping (Gaines \& Shaw, 1996), which might be proven valuable if combined by the unified modeling language (UML) (probably extended using its extension mechanisms).

Concluding, the positive answer to the question of whether it is feasible to aim at interoperation of LRBS for the automatic learning resources reusability and recreation depends on progress in conceptual, learning, social, and technological issues. The technological issues are the easiest to be solved. Consensus at conceptual, learning, and social levels is difficult to achieve but not impossible. Standardization can help, as well as research attempts along road maps, as the one published by Duval and Hodgins (2003).

\section{Acknowledgments}

The authors would like to acknowledge the support of the European Commission through grants HPRI-CT-1999-00026 (the TRACS Programme at EPCC) and the IST UNIVERSAL project. Many thanks to P. Avgeriou, P. Constantinou, I. Stavrou, and L. Michael for their reviews and valuable feedback on the draft versions of this chapter.

\section{References}

Douglas, I. W. (2001). Instructional design based on reusable learning objects: Applying lessons of object-oriented software engineering to learning systems design. Proceedings of the 31st ASEE/IEEE Frontiers in Education Conference, October 10-13, 2001. Reno, NV.

Downes, S. (2001). Learning objects: Resources for distance education worldwide. International Review of Research in Open and Distance Learn-

Copyright (C) 2005, Idea Group Inc. Copying or distributing in print or electronic forms without written permission of Idea Group Inc. is prohibited. 
ing, July.

Duncan, C. (2002). Digital repositories: The back office of e-learning or all learning. 9th International Conference ALT-C 2002: Learning technologies for communication, September 9-11. University of Sunderland, Sunderland.

Duval, E., \& Hodgins, W. (2003). A LOM research agenda. The 12th International World Wide Web Conference, May 20-24, 2003. Budapest, Hungary.

Gaines, B. R., \& Shaw, M. L. G. (1996). Web map: Concept mapping on the Web. Proceedings of the fourth international World Wide Web conference (Vol. 1, Issue 1). Retrieved from the World Wide Web: http:// www.w3j.com/1/gaines.134/paper/134.html

IEEE, Learning Technology Standards Committee (LTSC). (2001). Draft standard for learning object metadata (LOM), Draft 6.4, 2001.

IEEE. (1990) IEEE standard computer dictionary: A compilation of IEEE standard computer glossaries. New York: IEEE.

IMS. (2001). IMS digital repositories interoperability-Core functions information model. Revision: January 13, 2003.

Milstead, J., \& Feldman, S. (1999). Metadata: Cataloging by any other name... Retrieved from the World Wide Web: http://www.onlinemag.net/OL1999/ milstead1.html

Nejdl, W., Wolf, B., Qu, C., Decker, S., Sintek, M., Naeve, A., Nilsson, M., Palmer, M., \& Risch, T. (2000). Edutella: A P2P networking infrastructure based on RDF. WWW2002, May 7-11. Honolulu, Hawaii. (ACM 1-58113449-5/02/0005)

National Governors Association. (2001). The state of e-learning in the states, NGA report. Retrieved June 6, 2001 from the World Wide Web: http:// www.nga.org/cda/files/060601ELEARNING.pdf

Stammers, R., Carey, M., \& Astley, J. (1990). Task analysis. In J. Wilson, \& E. N. Corlet (Eds.), Evaluation of human work (Chapter 6). Bristol, PA: Taylor \& Francis.

Wigley, W. (1985). INPO/Industry job and task analysis efforts. Proceedings of the IEEE Third Conference on Human Factors and Power Plants.

Zlomislic, S., \& Bates, A. W. (2002). Assessing the costs and benefits of telelearning: A case study from the University of British Columbia. Reports from the NCE-Telelearning project entitled "Developing and Applying a Cost-Benefit Model for Assessing Telelearning," Telelearning Networks of Centers of Excellence (http://research.cstudies.ubc.ca/nce/ EDST565.pdf). 


\section{Part III}

Applications and

Case Studies 